\title{
Hydrogen-rich water alleviates the toxicities of different stresses to mycelial growth in Hypsizygus marmoreus
}

Jinjing Zhang ${ }^{1}$, Haibo Hao ${ }^{1,2}$, Mingjie Chen ${ }^{1}$, Hong Wang ${ }^{1}$, Zhiyong Feng ${ }^{1 *}$ and Hui Chen ${ }^{1 *}$

\begin{abstract}
In plants, hydrogen gas $\left(\mathrm{H}_{2}\right)$ enhances tolerance to several abiotic stresses, including salinity and heavy metals. However, the effect of $\mathrm{H}_{2}$ on fungal growth under different stresses remains largely unclear. In this study, hydrogen-rich water (HRW) was employed to characterize physiological roles and molecular mechanisms of $\mathrm{H}_{2}$ in the alleviation of three different stresses in basidiomycete Hypsizygus marmoreus. Our results showed that HRW treatment, of which the $\mathrm{H}_{2}$ concentration was $0.8 \mathrm{mM}$, significantly reduced the toxicities of $\mathrm{CdCl}_{2}, \mathrm{NaCl}$ and $\mathrm{H}_{2} \mathrm{O}_{2}$, leading to significantly improved mycelial growth and biomass. These beneficial effects could be attributed to a significantly decreased formation of malondialdehyde (MDA). Besides, HRW treatment significantly increased the activities of antioxidants (SOD, CAT and GR) as well as the gene expressions of these antioxidants (SOD, CAT, and GR) at the mRNA level. In vivo detection of reactive oxygen species (ROS), including $\mathrm{H}_{2} \mathrm{O}_{2}$ and $\mathrm{O}_{2}{ }^{-}$, as well as lipid peroxidation provided further evidence that HRW could significantly improve tolerances of $\mathrm{CdCl}_{2}, \mathrm{NaCl}$ and $\mathrm{H}_{2} \mathrm{O}_{2}$. Furthermore, pyruvate kinase was activated in the mycelia treated with $\mathrm{HRW}$, along with its induced gene expression, suggesting that HRW treatment enhanced the glucose metabolism. Taken together, our findings suggested that the usage of HRW could be an effective approach for contaminant detoxification in H. marmoreus, which was similar with the effects of HRW in plants, and such effects could be also beneficial in entire agricultural system.
\end{abstract}

Keywords: Hypsizygus marmoreus, Hydrogen gas, Oxidative stress, Mycelial growth

\section{Introduction}

Hypsizygus marmoreus (Peck.) Bigelow (Tricholomataceae), also known as bunashimeji and hon-shimeji, has been successfully and commercially cultivated in East Asia (Akavia et al. 2006). In China, H. marmoreus has become increasingly popular due to its mild, sweet, nutty flavor and crunchy texture as well as some physiologically beneficial components. Nowadays, the substrates used for culturing mushrooms from plants, such as sawdust, corncob and wheat bran, are usually contaminated. Contaminations

\footnotetext{
*Correspondence: feng_zy@yahoo.com; Huichen_js@aliyun.com ${ }^{1}$ National Research Center for Edible Fungi Biotechnology and Engineering, Key Laboratory of Applied Mycological Resources and Utilization, Ministry of Agriculture, Shanghai Key Laboratory of Agricultural Genetics and Breeding, Institute of Edible Fungi, Shanghai Academy of Agricultural Sciences, No. 1000, Jinqi Road, Fengxian District, Shanghai 201403, China

Full list of author information is available at the end of the article
}

will severely inhibit mushroom growth, which may reduce the fruit body production. If the contaminations, such as heavy metals, are accumulated in crop plants, they will also accumulate in the fruit body of mushroom, posing a severe threat to human health through food chains (Järup and Åkesson 2009; Podazza et al. 2012).

In fungi, the oxidative stress, at least in part, is caused by stimulated generation of reactive oxygen species (ROS), which is able to modify the antioxidant defense and elicit oxidative stress (Rodríguez-Serrano et al. 2006, 2009; Schützendübel et al. 2001). ROS, including * $\mathrm{OH}$, $\mathrm{O}_{2}^{-}$and $\mathrm{H}_{2} \mathrm{O}_{2}$, if uncontrolled, can cause oxidative damage to macromolecules, such as lipids, thus leading to lipid peroxidation and cell death (Bailly 2004). The enzymatic system, including superoxide dismutase (SOD), catalase (CAT), glutathione peroxidase (GPX) and guaiacol peroxidase (POD), can scavenge the ROS and enhance the fungal growth. 
Recently research has found that hydrogen gas $\left(\mathrm{H}_{2}\right)$ is a potentially 'novel' antioxidant in plants and animals. As the most abundant chemical element in the universe, $\mathrm{H}_{2}$ is a colorless, odorless, tasteless and highly combustible diatomic gas that has been known for many years (Huang et al. 2010). However, direct use of $\mathrm{H}_{2}$ is dangerous and flammable (Xie et al. 2012). Therefore, most of researchers use the hydrogen-rich water (HRW) to perform experiments, which is safe, cost-effective and commercially available. In plants and animals, HRW has been widely used as an antioxidant (Ohta 2012; Xie et al. 2012).

In animals, HRW, as an antioxidant, has beneficial effects in preventive and therapeutic applications, and such effects have been reported in 38 diseases and physiological states, including Parkinson, atherosclerosis, glaucoma and hepatic ischemia disease (Ohta 2012). In plants, HRW has been also used to illustrate that $\mathrm{H}_{2}$ can act as a novel beneficial gaseous molecule in plant adaptive responses (Cui et al. 2013; Jin et al. 2013; Wu et al. 2015a; Xie et al. 2012). In Chinese cabbage, HRW improves $\mathrm{CdCl}_{2}$ tolerance by reducing $\mathrm{CdCl}_{2}$ uptake and increasing antioxidant defense (Wu et al. 2015b). In Arabidopsis, HRW can enhance its salt tolerance by increasing antioxidant defense and significantly counteracting the $\mathrm{NaCl}$-induced ROS overproduction and lipid peroxidation (Xie et al. 2012). Under high light stress, $\mathrm{HRW}$ decreases the levels of $\mathrm{O}_{2}{ }^{-}$and $\mathrm{H}_{2} \mathrm{O}_{2}$ and elevates the activities of antioxidants, including SOD, CAT, APX and GR (Zhang et al. 2015a). Besides, HRW can activate $\alpha / \beta$-amylase activity, thus accelerating the formation of reducing sugar and total soluble sugar in rice (Xu et al. 2013). However, there is not report about the effects of HRW on the fungal growth in stressful environment.

In the present study, we evaluated the effects of HRW on the mycelial growth under three types of stresses $\left(\mathrm{CdCl}_{2}, \mathrm{NaCl}\right.$ and $\left.\mathrm{H}_{2} \mathrm{O}_{2}\right)$ and found that HRW treatment enhanced the mycelial growth and increased the mycelial biomass. In addition, HRW could enhance the antioxidant activities, decreased the ROS level and alleviated the lipid peroxidation in mycelia of $H$. marmoreus. Moreover, $\mathrm{HRW}$ also activated the pyruvate kinase (PK) in the mycelia. These results suggested a positive role of HRW in reducing pollutant residues for mushroom safety.

\section{Materials and methods}

\section{Fungal materials, growth conditions, experimental design} and growth analysis

Hypsizygus marmoreus samples were obtained from the China General Microbiological Culture Collection Center (Beijing) (No. CGMCC5.01974). First, the mycelium of $\mathrm{H}$. marmoreus was cultured on PDA medium at $25{ }^{\circ} \mathrm{C}$ for 2 weeks, and then it was transferred onto PDB and PDA medium containing $\mathrm{CdCl}_{2}, \mathrm{NaCl}$ or $\mathrm{H}_{2} \mathrm{O}_{2}$ of different concentrations. The mycelial biomass was determined on PDB medium, and the mycelial growth was examined on PDA medium under various stresses. The $50 \%$ inhibition concentrations of $\mathrm{CdCl}_{2}, \mathrm{NaCl}$ and $\mathrm{H}_{2} \mathrm{O}_{2}$ were also evaluated accordingly.

Besides, the mycelia were transferred into solutions containing 0 or $50 \%$ inhibition concentration of $\mathrm{CdCl}_{2}$, $\mathrm{NaCl}$ and $\mathrm{H}_{2} \mathrm{O}_{2}$ and then incubated at $25{ }^{\circ} \mathrm{C}$ for $24 \mathrm{~h}$. Subsequently, the mycelia were transferred into HRW for 5 days, and the HRW was replaced every $12 \mathrm{~h}$. Mycelia without HRW treatment were used as the control $\left(\mathrm{H}_{2} \mathrm{O}\right)$. These above-mentioned treatments could be described as follows: (1) $\mathrm{H}_{2} \mathrm{O} \rightarrow \mathrm{H}_{2} \mathrm{O}, \mathrm{H}_{2} \mathrm{O} \rightarrow \mathrm{HRW}, \mathrm{CdCl}_{2} \rightarrow \mathrm{H}_{2} \mathrm{O}$, $\mathrm{CdCl}_{2} \rightarrow$ HRW, $\mathrm{H}_{2} \mathrm{O}_{2} \rightarrow \mathrm{H}_{2} \mathrm{O}, \mathrm{H}_{2} \mathrm{O}_{2} \rightarrow$ HRW, $\mathrm{NaCl} \rightarrow \mathrm{H}_{2} \mathrm{O}, \mathrm{NaCl} \rightarrow$ HRW. In addition, the mycelial growth was determined after the HRW treatment for 5 days. Meanwhile, the mycelial biomass was determined on PDB medium in the similar experimental treatment. Before being added to the medium, $\mathrm{CdCl}_{2}, \mathrm{NaCl}$ or $\mathrm{H}_{2} \mathrm{O}_{2}$ solutions were sterilized by filtration through a $0.22-\mu \mathrm{m}$ membrane. Co-treatment with HRW was applied on the 8th day, and 0 or $50 \%$ inhibition concentration of $\mathrm{CdCl}_{2}$, $\mathrm{NaCl}$ or $\mathrm{H}_{2} \mathrm{O}_{2}$ were added on the 9th day and then incubated at $25{ }^{\circ} \mathrm{C}$ for $24 \mathrm{~h}$ respectively. Mycelia were harvested and washed with a large amount of distilled water and then dried at $60^{\circ} \mathrm{C}$ to a constant biomass. The Growth tests were performed in triplicate. Ruler $(0.1 \mathrm{~cm})$ and electronic balance $(0.0001 \mathrm{~g})$ were used in measurements of mycelial growth and biomass after various treatments.

\section{Determination of $\mathrm{H}_{\mathbf{2}}$ concentrations}

HRW was kindly supplied by Beijing Hydrovita Beverage Co., Ltd. (Beijing, China) and the $\mathrm{H}_{2}$ concentration in the freshly HRW was $1.0 \mathrm{mM}$ at a hermetical canister. While the canister was opened, the $\mathrm{H}_{2}$ concentration was $0.8 \mathrm{mM}$ in $30 \mathrm{~min}$. The $\mathrm{H}_{2}$ concentration was maintained at a relative constant level in $25{ }^{\circ} \mathrm{C}$ for at least $12 \mathrm{~h}$. The $\mathrm{H}_{2}$ concentration was analyzed by using gas chromatography (GC). The chromatographic system (GC 7890, Agilent) was according the method described by Wu et al. (2015a) which was equipped with thermal conductivity detector (TCD). The working conditions were optimized as TCD detector temperature at $100{ }^{\circ} \mathrm{C}, 5 \AA$ molecular sieves as fixed phase, column temperature at $150^{\circ} \mathrm{C}$, oven temperature at $60{ }^{\circ} \mathrm{C}$. Nitrogen gas was used as carrier gas and air pressure $0.2 \mathrm{MPa}$.

\section{Histochemical detection of ROS}

$\mathrm{H}_{2} \mathrm{O}_{2}$ or $\mathrm{O}_{2}{ }^{-}$level was measured by 3, 3, 9-diaminobenzidine (DAB) or nitroblue tetrazolium (NBT) staining, respectively. First, the mycelia were cultured on PDB medium at $25{ }^{\circ} \mathrm{C} 150 \mathrm{rpm}$ for 10 days, and then the mycelia became mycelial pellet. Subsequently, different 
stresses were added into the PDB medium to make the PDB medium contained the $50 \mu \mathrm{M} \mathrm{CdCl}_{2}, 1 \% \mathrm{NaCl}$ or $2 \mathrm{mM} \mathrm{H}_{2} \mathrm{O}_{2}$ respectively. After the mycelia were cultured for $24 \mathrm{~h}$, the mycelial pellets were collected and transferred into $\mathrm{ddH}_{2} \mathrm{O}$ or HRW for $48 \mathrm{~h}$. Finally, the mycelial pellets were immersed in freshly prepared $\mathrm{DAB}$ solution $(0.1 \% \mathrm{w} / \mathrm{v}, \mathrm{pH} 3.8)$, vacuum-infiltrated, and then incubated at $25^{\circ} \mathrm{C}$ for $2 \mathrm{~h}$ in darkness. Alternatively, the mycelial pellets were immersed in NBT solution consisting of $10 \mathrm{mM}$ potassium phosphate (pH 7.8) and $10 \mathrm{mM}$ $\mathrm{NaN}_{3}$, vacuum-infiltrated, and then incubated at $25{ }^{\circ} \mathrm{C}$ for $1 \mathrm{~h}$ in darkness. After extensive wash, all the decolorized mycelial pellets were examined on a color film (Powershot G16; Canon Photo Film, Tokyo, Japan).

\section{Detection of $\mathrm{H}_{2} \mathrm{O}_{2}$ and MDA concentrations}

The organism produces oxygen free radicals through enzyme system and non-enzyme system. $\mathrm{H}_{2} \mathrm{O}_{2}$ is the product of the enzyme system. Fresh mycelia (1.0 g) were homogenized in a mortar with $10 \mathrm{~mL}$ physiological saline on ice. Then, the obtained $10 \%$ homogenate was centrifuged at $2500 \mathrm{~g}$ for $10 \mathrm{~min}$. The supernatant was used to detect the concentration of $\mathrm{H}_{2} \mathrm{O}_{2}$, which was analyzed using Hydrogen Peroxide assay kit (NJJCBio. Ltd., China). The absorbance of the supernatant was read at a wavelength of $405 \mathrm{~nm}$, and $\mathrm{dd}_{2} \mathrm{O}$ was used as the blank.

$\mathrm{H}_{2} \mathrm{O}_{2}$ can induce the lipid peroxidation by attacking poly unsaturated fatty acid in the biological membrane and then form lipoid peroxide, injuring the cells and tissue. The malondialdehyde (MDA) was used as an indicator of oxidative stress in non-enzyme system. The supernatant of $10 \%$ homogenate was also used to detect the concentration of MDA, which was determined by MDA assay kit (TBA method) (NJJCBio. Ltd., China). The absorbance of the supernatant was read at a wavelength of $532 \mathrm{~nm}$, and $\mathrm{ddH}_{2} \mathrm{O}$ was used as the blank.

\section{Antioxidant activity assays}

Fresh mycelia $(1.0 \mathrm{~g})$ were homogenized in $9.0 \mathrm{~mL}$ of $0.1 \mathrm{M}$ phosphate buffer ( $\mathrm{PH} 7.0)$ on ice. Then, the $10 \%$ homogenate was centrifuged at $4000 \mathrm{~g}$ for $10 \mathrm{~min}$ at $4{ }^{\circ} \mathrm{C}$, and the supernatant was used for assays of superoxide dismutase (SOD), catalase (CAT) and glutathione reductase (GR) activities. The CAT activity was detected according to the instruction of Catalase (CAT) assay kit (Visible light) (Nanjing Jiancheng Bioengineering Institute, Nanjing, China). One unit of CAT was defined as the mount of enzyme for decomposing $1 \mu \mathrm{mol} \mathrm{H}_{2} \mathrm{O}_{2}$ monitored at $405 \mathrm{~nm}$. The SOD activity was detected as description by Total Superoxide Dismutase (T-SOD) assay kit (Hydroxylamine method) (Nanjing Jiancheng Bioengineering Institute, Nanjing, China). One unit of SOD activity was defined as the amount of SOD while inhibition of SOD was up to $50 \%$ per gram tissue in $1 \mathrm{~mL}$ reacted solution and the SOD activity was monitored at $550 \mathrm{~nm}$. The GR activity was detected as description by glutathione reductases assay kit (Nanjing Jiancheng Bioengineering Institute, Nanjing, China) monitored at $340 \mathrm{~nm}$.

\section{Total protein detection}

The protein concentration of mycelia used for calculating was detected as described by Total protein quantitative assay kit (Coomassie Brilliant Blue) (Nanjing Jiancheng Bioengineering Institute, Nanjing, China) monitored at $595 \mathrm{~nm}$. The standard protein concentration used in this assay kit was $0.563 \mathrm{~g} / \mathrm{L}$ and the color solution was the coomassie brilliant solution.

\section{Determination of $\mathrm{CdCl}_{2}$ and $\mathrm{NaCl}$ content in mycelia}

The concentrations of $\mathrm{CdCl}_{2}$ were determined using an atomic absorption spectrophotometer (180-80 Hitachi, Tokyo, Japan) as described by Liu et al. (2008). The concentration of $\mathrm{NaCl}$ element was measured by an Inductively Coupled Plasma-Optical Emission Spectrometer (ICPOES, Perkin Elmer Optima 2100DV) by using the total sodium content kit (Comin Biotechnology, Suzhou, China).

\section{Real-time quantitative RT-PCR analysis}

Total RNA was isolated from fresh mycelia using Trizol reagent (Takara, Dalian, China) according to the manufacturer's instructions. The RNA purity was verified based on the ratio $(>2.0)$ of $260 / 280 \mathrm{~nm}$ using the gDNA assay kit (Takara, Dalian, China). Subsequently, $20 \mu \mathrm{L}$ purified RNA was reversely transcribed into cDNA in a 40- $\mu \mathrm{L}$ reaction system according to the manufacturer's instructions. Real-time quantitative PCR reactions were performed as described by Zhang et al. (2014, 2015a, b) using SYBR (Takara, Dalian, China). Additional file 1: Table S1 lists the primer sequences and accession numbers of the target genes, and $18 \mathrm{~S}$ ribosomal RNA was selected as the housekeeping gene in the present study. Each experiment was performed in triplicate. The relative gene expression was analyzed using the $2^{-\Delta \Delta \mathrm{Ct}}$ method described by Livak and Schmittgen (2001).

\section{Data presentation and statistical analysis}

Values are shown as the mean \pm SD of three independent experiments with three replicates each. Differences among treatments were analyzed by one-way analysis of variance (ANOVA) combined with Duncan's multiple range test at a probability of $\mathrm{P}<0.05$.

\section{Results}

Effects of $\mathrm{CdCl}_{2}, \mathrm{NaCl}$ and $\mathrm{H}_{2} \mathrm{O}_{2}$ on the mycelial growth To detect the sensitivity of $H$. marmoreus to $\mathrm{CdCl}_{2}, \mathrm{NaCl}$ and $\mathrm{H}_{2} \mathrm{O}_{2}$, mycelia were cultured on the PDA and PDB medium 
with different concentrations of $\mathrm{CdCl}_{2}, \mathrm{NaCl}$ and $\mathrm{H}_{2} \mathrm{O}_{2}$. The concentrations of $\mathrm{CdCl}_{2}$ were 50,100 and $150 \mu \mathrm{M}$, the concentrations of $\mathrm{NaCl}$ were $0.5,1$ and $2 \%$, and the concentrations of $\mathrm{H}_{2} \mathrm{O}_{2}$ were 1,2 and $4 \mathrm{mM}$. Figure 1 shows that the mycelial growth was markedly inhibited by the three types of stresses, exhibiting significantly decreased mycelial growth (Fig. 1a) and mycelial biomass (Fig. 1b). Therefore, $50 \mu \mathrm{M}$ $\mathrm{CdCl}_{2}, 1 \% \mathrm{Na}$ and $2 \mathrm{mM} \mathrm{H}_{2} \mathrm{O}_{2}$ were subsequently used to investigate the role of $\mathrm{HRW}$ in the alleviation of inhibitory effects of the three stresses on mycelial growth.

\section{HRW alleviates the inhibitory effects of $\mathrm{CdCl}_{2}, \mathrm{NaCl}$ and $\mathrm{H}_{2} \mathrm{O}_{2}$ on mycelial growth}

To evaluate the effects of HRW on the mycelial growth under three different stresses, we placed mycelial pellets

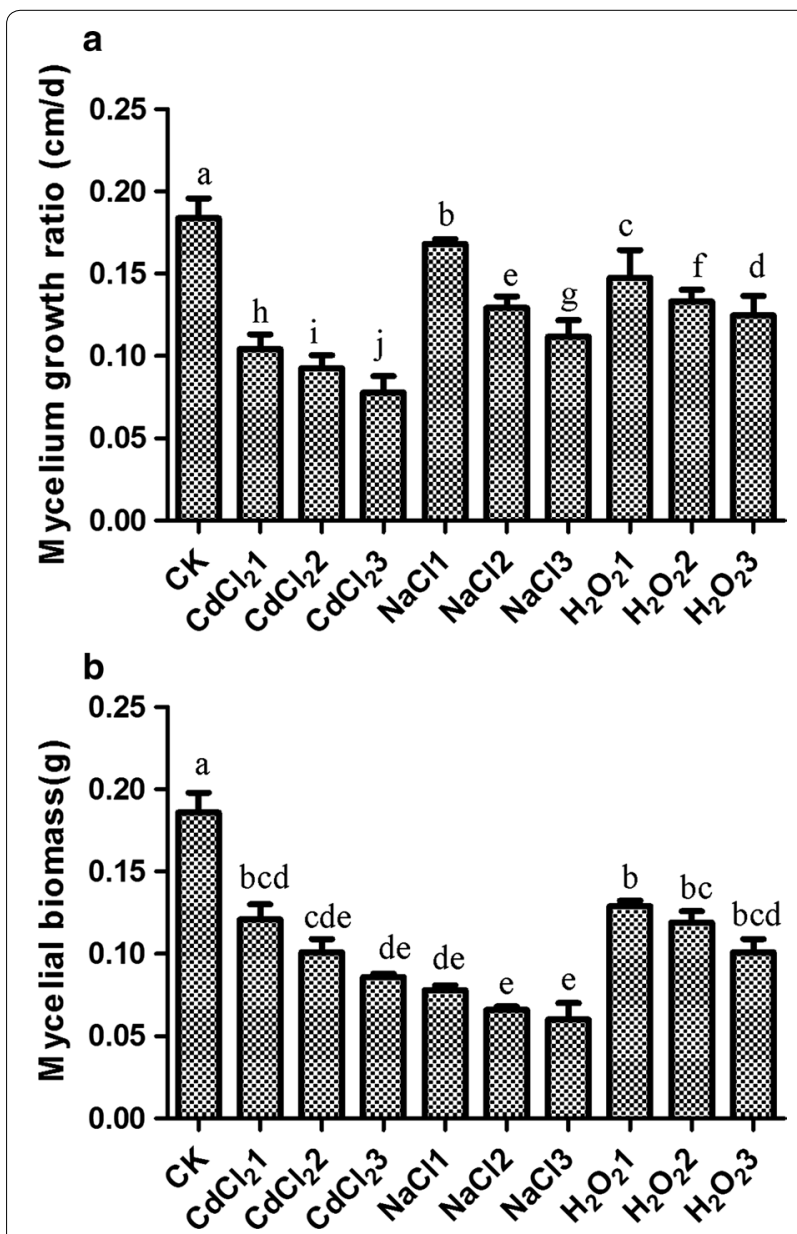

Fig. 1 Effects of different concentrations of $\mathrm{CdCl}_{2}, \mathrm{NaCl}$ and $\mathrm{H}_{2} \mathrm{O}_{2}$ on mycelial growth and biomass of Hypsizygus marmoreus. Mycelia were incubated with different concentrations of $\mathrm{CdCl}_{2}, \mathrm{NaCl}$ and $\mathrm{H}_{2} \mathrm{O}_{2}$. The mycelial growth was detected on PDA medium after 10 days of incubation (a), and the mycelial biomass was detected on PDB medium after 10 days of incubation $(\mathbf{b})$. Data are mean \pm SE of three independent experiments. Bars with different letters are significantly different at $P<0.05$ according to Duncan's multiple range test in the solutions for $24 \mathrm{~h}$ and then transferred them into solutions with $\mathrm{H}_{2} \mathrm{O}$ or HRW for 5 days. During this process, HRW was replaced every $12 \mathrm{~h}$. The mycelial growth was determined after 5 days of cultivation. Figure $2 \mathrm{a}$ and c display that the HRW treatment could enhance the mycelial growth by alleviating the toxicities of $\mathrm{CdCl}_{2}$, $\mathrm{NaCl}$ and $\mathrm{H}_{2} \mathrm{O}_{2}$ to mycelia (Fig. 2a, c).

Besides, the effects of HRW on the mycelial biomass under three different stresses were assessed on PDB medium. If the three stresses were added at the early stage of cultivation, the mycelia could not grow and died finally. Therefore, after 10 days of mycelial culture, the three stresses $\left(50 \mu \mathrm{M} \mathrm{CdCl}_{2}, 1.0 \% \mathrm{NaCl}\right.$ or $\left.2 \mathrm{mM} \mathrm{H}_{2} \mathrm{O}_{2}\right)$ were respectively added into the PDB medium, and the culture was maintained for another $24 \mathrm{~h}$. The stresses were then removed, and the mycelia were transferred into HRW for 5 days. Subsequently, mycelia were collected and dried at $65{ }^{\circ} \mathrm{C}$ until the biomass was stable. Figure $2 \mathrm{~b}$ shows that the HRW treatment could significantly increase the mycelial biomass compared with the control (CK) group. Besides, the HRW treatment significantly slowed down the accumulation of $\mathrm{CdCl}_{2}, \mathrm{H}_{2} \mathrm{O}_{2}$ and $\mathrm{NaCl}$. The $\mathrm{CdCl}_{2}, \mathrm{NaCl}$ and $\mathrm{H}_{2} \mathrm{O}_{2}$ contents in the mycelia was 20.08, 9.89 and $30.39 \%$ lower than the $\mathrm{CdCl}_{2}$, $\mathrm{NaCl}$ and $\mathrm{H}_{2} \mathrm{O}_{2}$-stressed alone mycelia (Table 1).

\section{HRW enhances the antioxidant activities of mycelia}

After the mycelia were cultured on PDB medium containing $50 \mu \mathrm{M} \mathrm{CdCl}_{2}, 1 \% \mathrm{NaCl}$ or $2 \mathrm{mM} \mathrm{H}_{2} \mathrm{O}_{2}$ for $24 \mathrm{~h}$, the activities of several antioxidants, including CAT, SOD and GR, were determined. Figure 3 shows the sensitivity of the three antioxidants to $\mathrm{CdCl}_{2}, \mathrm{NaCl}$ and $\mathrm{H}_{2} \mathrm{O}_{2}$. The SOD activity was significantly inhibited by $\mathrm{CdCl}_{2}$, $\mathrm{NaCl}$ and $\mathrm{H}_{2} \mathrm{O}_{2}$. In contrast, the activities of CAT and GR were significantly induced by $\mathrm{CdCl}_{2}, \mathrm{NaCl}$ and $\mathrm{H}_{2} \mathrm{O}_{2}$. The pretreated mycelia were transferred into $\mathrm{H}_{2} \mathrm{O}$ or HRW for 5 days, and the mycelia were used to determine the enzyme activities of the three antioxidants. Figure 4 reveals that the enzyme activities of the three antioxidants were restored after the HRW treatment compared with the CK group, indicating that the HRW treatment induced the activities of the antioxidants. Moreover, higher CAT and GR activities were detected after the co-treatment with HRW compared with pretreatment of $\mathrm{CdCl}_{2}, \mathrm{NaCl}$ and $\mathrm{H}_{2} \mathrm{O}_{2}$ alone.

\section{HRW alleviates ROS homeostasis and lipid peroxidation}

The effects of HRW on the ROS production induced by $\mathrm{CdCl}_{2}, \mathrm{NaCl}$ and $\mathrm{H}_{2} \mathrm{O}_{2}$ were investigated by histochemical straining, including NBT staining for $\mathrm{O}^{2-}$ and $\mathrm{DAB}$ staining for $\mathrm{H}_{2} \mathrm{O}_{2}$. The mycelia were cultured on PDB medium containing $\mathrm{CdCl}_{2}, \mathrm{NaCl}$ and $\mathrm{H}_{2} \mathrm{O}_{2}$ for $24 \mathrm{~h}$ and then transferred into $\mathrm{ddH}_{2} \mathrm{O}$ or HRW for 5 days. Figure 5 


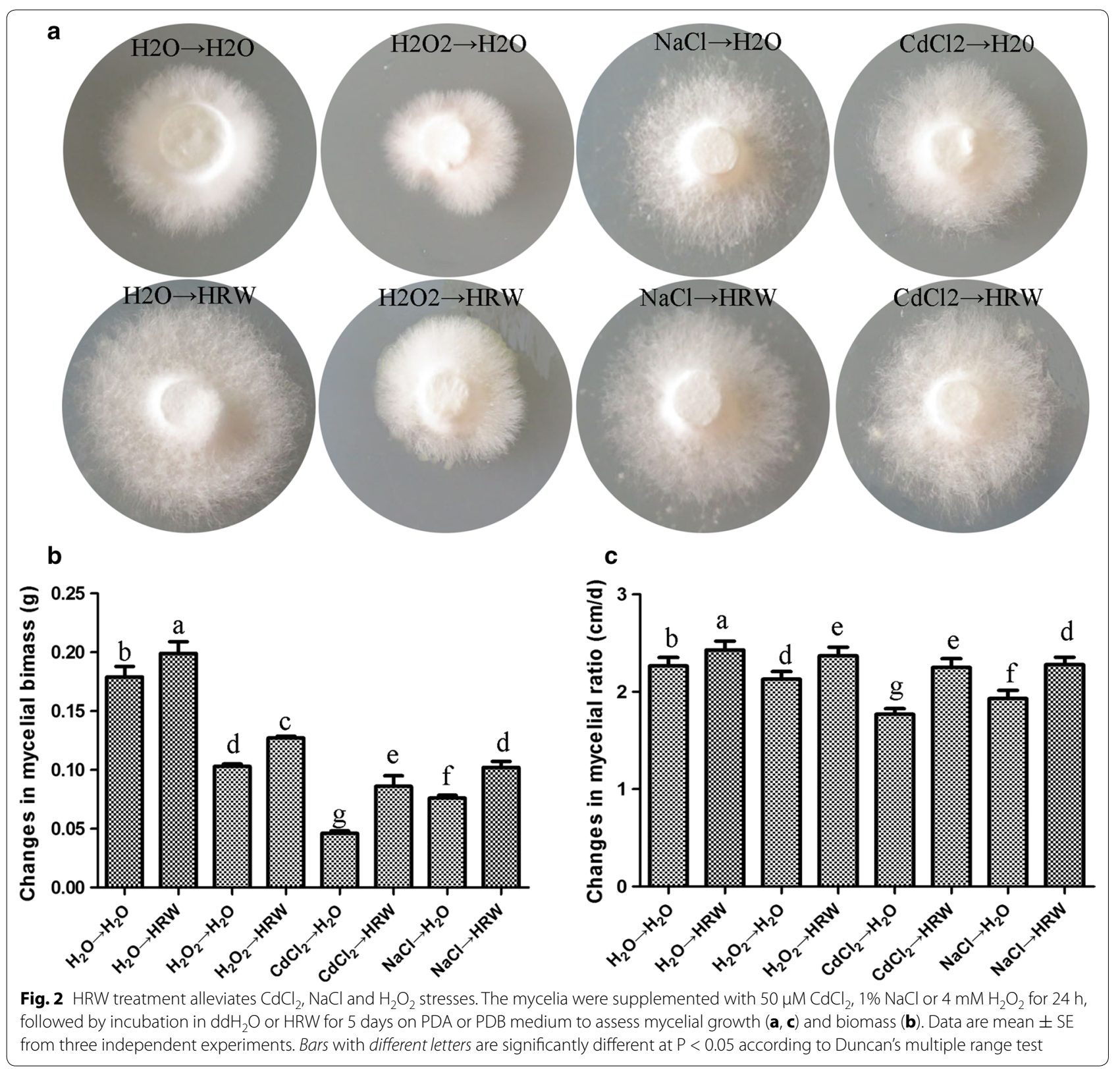

exhibits that low levels of $\mathrm{H}_{2} \mathrm{O}_{2}$ (DAB staining) (Fig. 5a) and $\mathrm{O}_{2}{ }^{-}$(NBT staining) (Fig. 5b) were detected in the $\mathrm{HRW}$-treated mycelia. However, $\mathrm{ddH}_{2} \mathrm{O}$-treated mycelia had higher production of $\mathrm{H}_{2} \mathrm{O}_{2}$ (DAB staining) and $\mathrm{O}_{2}{ }^{-}$ (NBT staining) compared with the HRW-treated mycelia. Besides, the levels of $\mathrm{H}_{2} \mathrm{O}_{2}$ were also detected using the $\mathrm{H}_{2} \mathrm{O}_{2}$ detection assay kit. Figure $5 \mathrm{c}$ shows that the levels of $\mathrm{H}_{2} \mathrm{O}_{2}$ in HRW-treated mycelia were lower compared with the $\mathrm{ddH}_{2} \mathrm{O}$-treated mycelia. These results were consistent with the DAB staining.

In addition, the MDA formation, which is a reliable marker of lipid peroxidation and free radical generation, was examined to clarify whether the beneficial effects of HRW were related to oxidative stress. After 24-h pretreatment of $\mathrm{CdCl}_{2}, \mathrm{NaCl}$ and $\mathrm{H}_{2} \mathrm{O}_{2}$, mycelia were transferred into $\mathrm{ddH}_{2} \mathrm{O}$ or HRW for another $48 \mathrm{~h}$. As expected, the $\mathrm{ddH}_{2} \mathrm{O}$ treatment caused significantly increased MDA content (Fig. 5d). However, the HRW treatment triggered a significant reduction in MDA level.

\section{HRW enhances the PK activity}

As HRW could enhance the mycelial growth by reducing the oxidative stress, we detected the effect of HRW on the activity of $\mathrm{PK}$, which is an important enzyme in 
Table 1 Effects of $\mathrm{HRW}$ treatment on $\mathrm{CdCl}_{2}, \mathrm{NaCl}$ and $\mathrm{H}_{2} \mathrm{O}_{2}$ concentrations in mycelia of $\mathrm{H}$. marmoreus

\begin{tabular}{|c|c|c|c|}
\hline Treatments & $\mathrm{CdCl}_{2}(\mathrm{nmol} / \mathrm{L})$ & $\mathrm{H}_{2} \mathrm{O}_{2}(\mathrm{mmol} / \mathrm{g})$ & $\mathrm{NaCl}(\mathrm{mg} / \mathrm{g})$ \\
\hline $\mathrm{H}_{2} \mathrm{O} \rightarrow \mathrm{H}_{2} \mathrm{O}$ & $N D^{c}$ & $62.4 \pm 4.328^{c}$ & $0.101 \pm 0.008^{c}$ \\
\hline $\mathrm{H}_{2} \mathrm{O} \rightarrow \mathrm{HRW}$ & $N D^{c}$ & $60.1 \pm 6.301^{c}$ & $0.098 \pm 0.003^{c}$ \\
\hline $\mathrm{CdCl}_{2} \rightarrow \mathrm{H}_{2} \mathrm{O}$ & $230.78 \pm 9.876^{\mathrm{a}}$ & - & - \\
\hline $\mathrm{CdCl}_{2} \rightarrow \mathrm{HRW}$ & $184.62 \pm 11.752^{b}$ & - & - \\
\hline $\mathrm{NaCl} \rightarrow \mathrm{H}_{2} \mathrm{O}$ & - & - & $2.307 \pm 0.506^{a}$ \\
\hline $\mathrm{NaCl} \rightarrow \mathrm{HRW}$ & - & - & $1.606 \pm 0.340^{b}$ \\
\hline $\mathrm{H}_{2} \mathrm{O}_{2} \rightarrow \mathrm{H}_{2} \mathrm{O}$ & - & $132.18 \pm 9.672^{\mathrm{a}}$ & - \\
\hline $\mathrm{H}_{2} \mathrm{O}_{2} \rightarrow \mathrm{HRW}$ & - & $119.11 \pm 11.012^{b}$ & - \\
\hline
\end{tabular}

8-day-old mycelia were pretreated with $0,50 \mu \mathrm{m} \mathrm{CdCl}, 1.0 \% \mathrm{NaCl}$ and $2 \mathrm{mM}$ $\mathrm{H}_{2} \mathrm{O}_{2}$ for $24 \mathrm{~h}$ and then were treated with or without treatment with $100 \%$ HRW. The control group was treated with water. Values are mean \pm SE of three independent experiments with at least three replicates for each. Different letters within columns indicate significant differences $(P<0.05)$ according to Duncan's multiple range test

reduced sugar metabolism. The mycelia were cultured on PDB medium for 10 days, and then the three stresses were added to the medium for $24 \mathrm{~h}$. We found that the PK activity was increased by 1.8-, 5.5- and 4.0 folds by addition of $\mathrm{CdCl}_{2}, \mathrm{NaCl}$ and $\mathrm{H}_{2} \mathrm{O}_{2}$, respectively (Fig. 6a). After $24 \mathrm{~h}$ of stress pretreatment, the mycelia were transferred into HRW for $48 \mathrm{~h}$. Figure $6 \mathrm{~b}$ shows that the PK activity was increased in the HRW-treated mycelia compared with the CK group. Besides, the expression of PK at the mRNA level was also induced by the HRW treatment.

\section{Gene expression analysis by qRT-PCR}

To confirm the above-mentioned findings, we examined the expressions of genes encoding SOD, CAT, GR and PK. The $18 \mathrm{~s}$ ribosomal RNA was used as a housekeeping gene, and the $\mathrm{dd}_{2} \mathrm{O}$-treated mycelia under the stressed conditions were used as the CK samples. Figure 7 reveals that the expressions of the above-mentioned four genes were all increased after the HRW treatment. In the $\mathrm{CdCl}_{2}$ pretreatment, the expressions of the four genes were induced by the HRW treatment at the lowest level. CAT most sensitively responded to the $\mathrm{CdCl}_{2}$ pretreatment. In the $\mathrm{Na}$ pretreatment, the expressions of the four genes were induced by the HRW treatment at the highest level. SOD most sensitively responded to the Na pretreatment, and its expression level was increased by 135 folds compared with the CK group. In the $\mathrm{H}_{2} \mathrm{O}_{2}$ pretreatment, GR most sensitively responded to the pretreatment, showing an increased expression of 37.5 folds.

\section{Discussion}

Hypsyzigus marmoreus is one of commercial mushrooms. In 2012, the daily production of H. marmoreus was 167 tons in China (Zhang et al. 2016a) according to data from

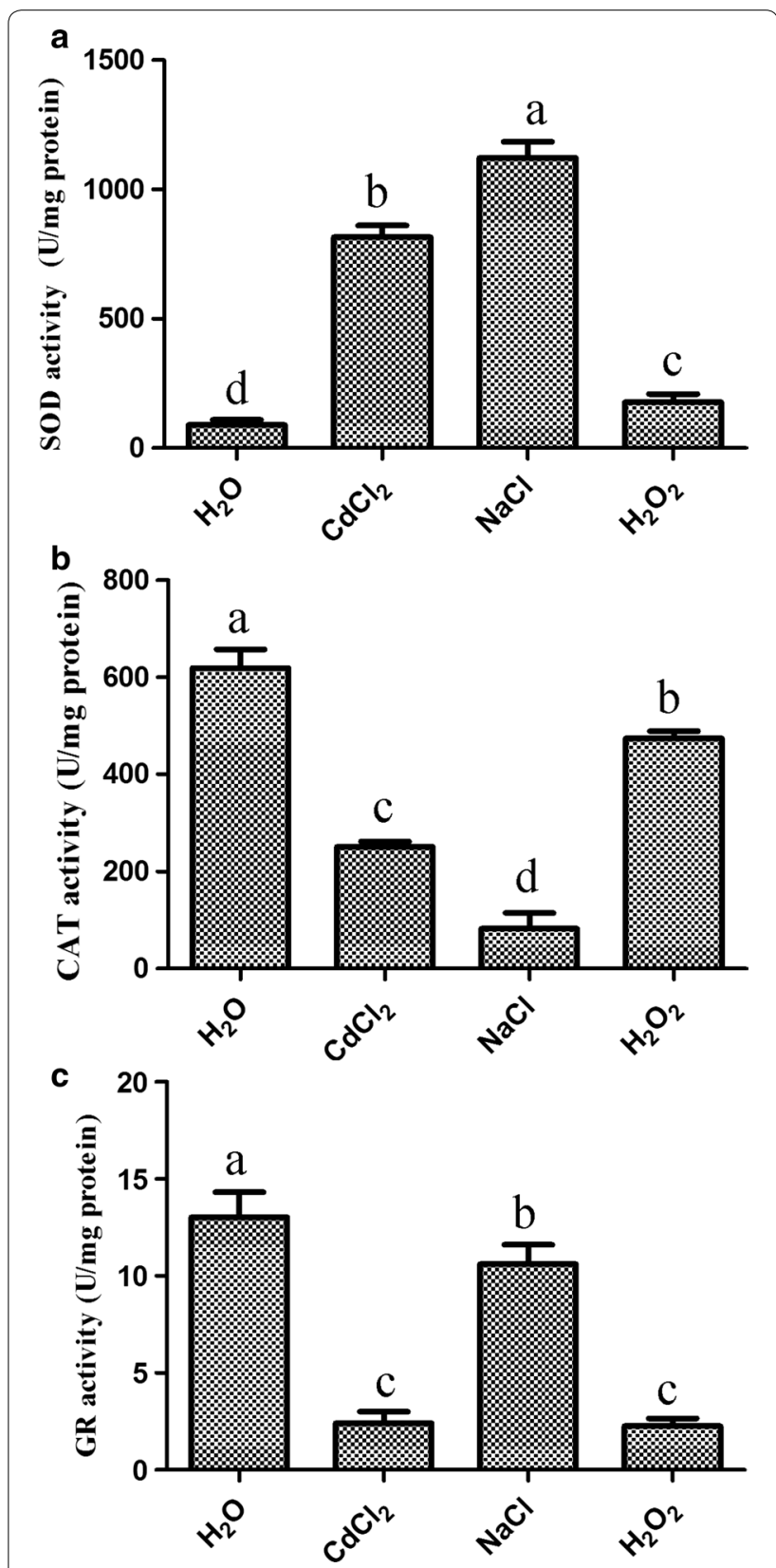

Fig. 3 Effects of $\mathrm{CdCl}_{2}, \mathrm{NaCl}$ and $\mathrm{H}_{2} \mathrm{O}_{2}$ treatments on SOD (a), CAT (b) and GR (c) activities in mycelia of Hypsizygus marmoreus. The mycelia were cultured on PDB medium for 10 days, followed by $24 \mathrm{~h}$ incubation with $\mathrm{CdCl}_{2}, \mathrm{NaCl}$ and $\mathrm{H}_{2} \mathrm{O}_{2}$. Data are mean $\pm \mathrm{SE}$ from three independent experiments. Bars with different letters are significantly different at $P<0.05$ according to Duncan's multiple range test

the China Edible Fungi Association, suggesting the rapidly increased demand for H. marmoreus and consumption of substrates. In edible mushrooms, they are usually grown on agricultural wastes which may contain toxic substances, such as heavy metals. These adverse factors may inhibit the mushroom growth and affect the fruit 


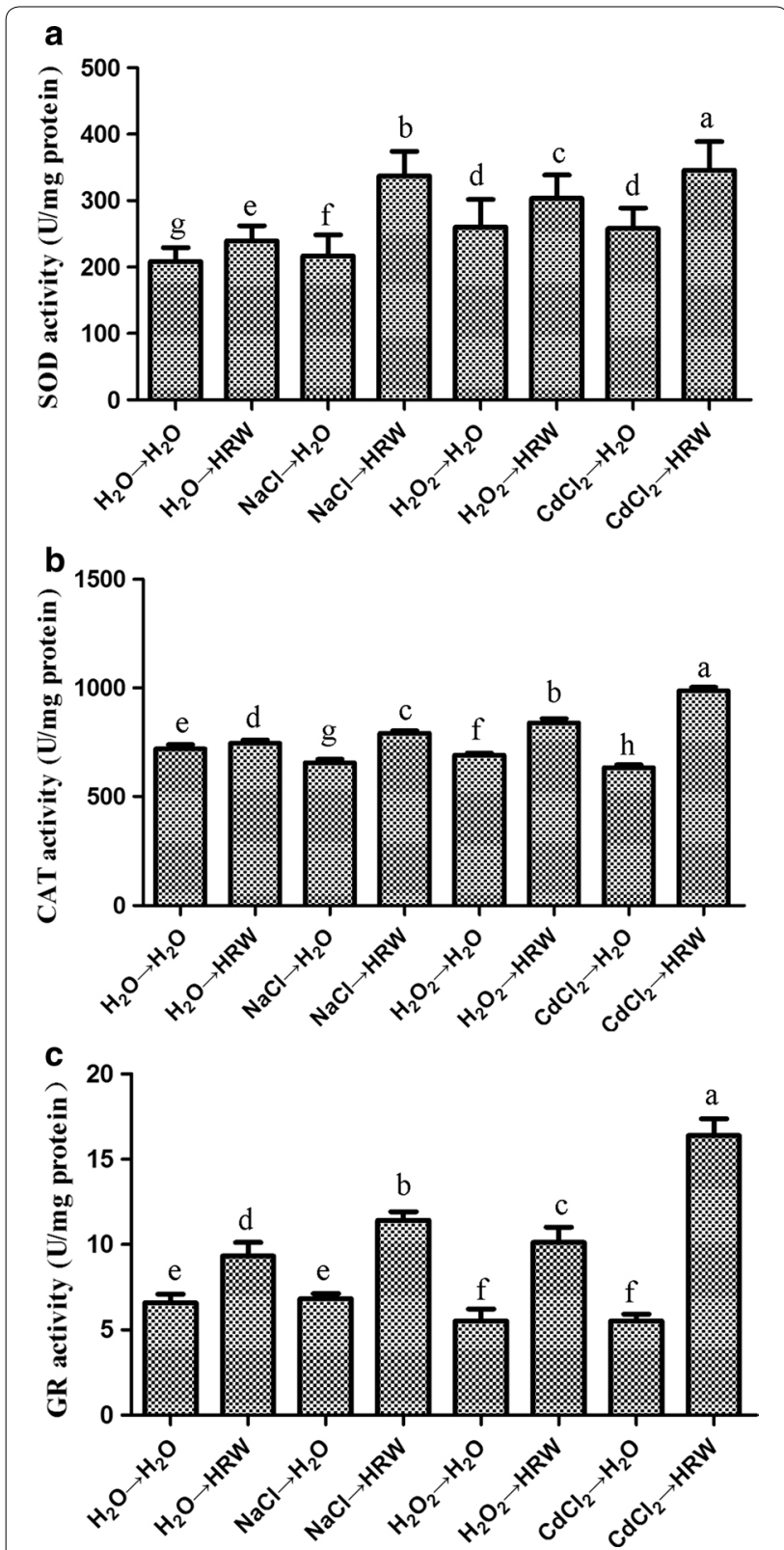

Fig. 4 Effects of HRW treatment on SOD (a), CAT (b) and GR (c) activities in mycelia of Hypsizygus marmoreus upon $\mathrm{CdCl}_{2}, \mathrm{NaCl}$ and $\mathrm{H}_{2} \mathrm{O}_{2}$ stresses. The mycelia were pretreated with $\mathrm{CdCl}_{2}, \mathrm{NaCl}$ or $\mathrm{H}_{2} \mathrm{O}_{2}$ for $24 \mathrm{~h}$, followed by 48 -h incubation with or without HRW. Data are mean \pm SE from three independent experiments. Bars with different letters are significantly different at $\mathrm{P}<0.05$ according to Duncan's multiple range test

body production. In mushrooms, many previous studies have reported the effects of heavy metals and $\mathrm{H}_{2} \mathrm{O}_{2}$ on the growth of edible mushrooms (Hatvani and Mecs 2003; Zharare et al. 2010).

In this present study, we first assessed the effects of $\mathrm{CdCl}_{2}, \mathrm{NaCl}$ and $\mathrm{H}_{2} \mathrm{O}_{2}$ at different concentrations on the

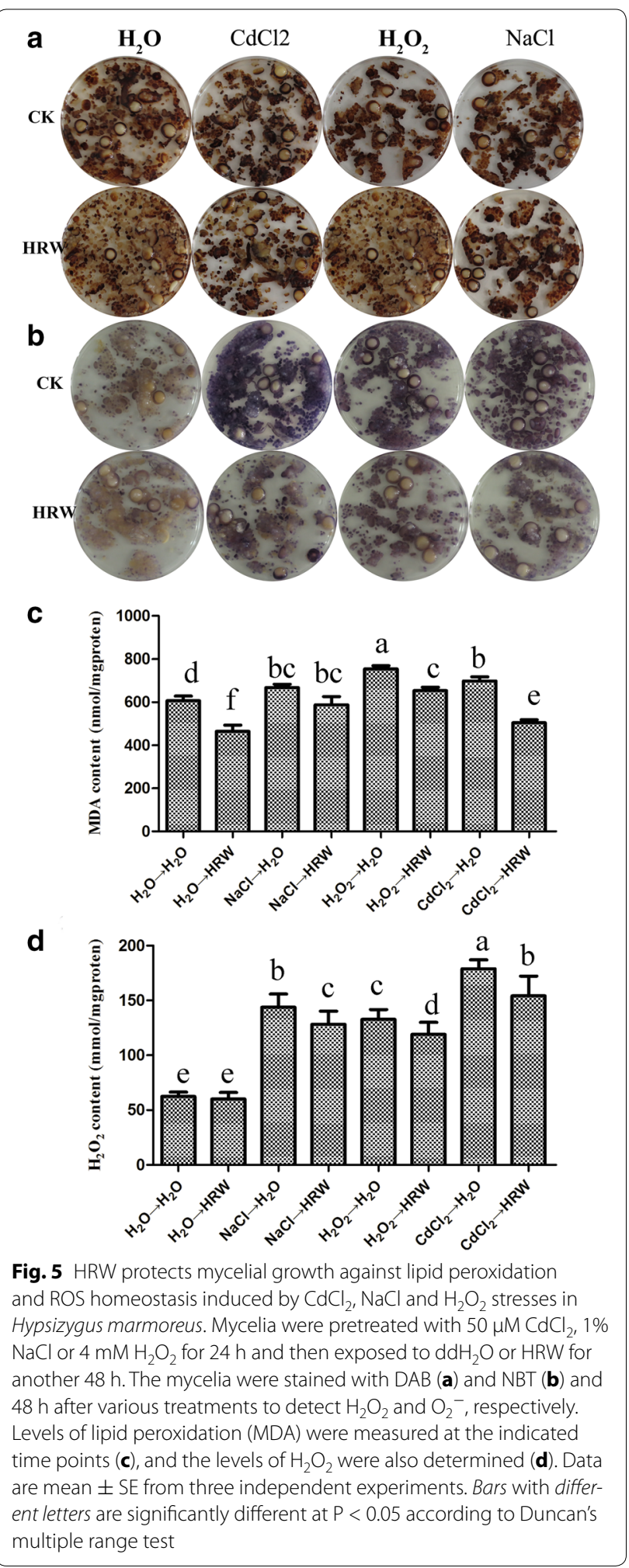

mycelial growth of $H$. marmoreus. $\mathrm{CdCl}_{2}(50 \mu \mathrm{M}), \mathrm{NaCl}$ (1.0\%) and $\mathrm{H}_{2} \mathrm{O}_{2}(2 \mathrm{mM})$ could cause about $50 \%$ reduction in mycelial growth in solid medium. The mycelia 


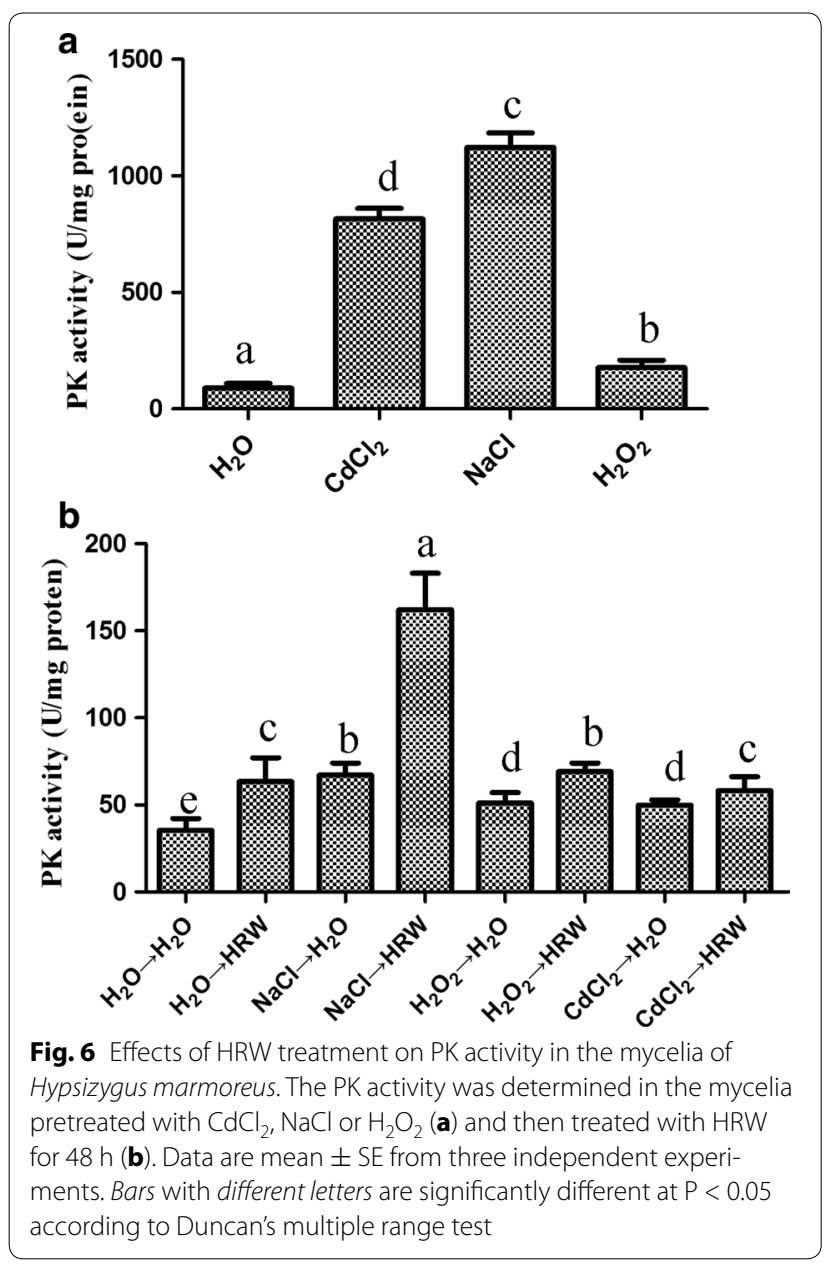

became thinner compared with the control as the concentrations of $\mathrm{CdCl}_{2}$ and $\mathrm{NaCl}$ were increased. In contrast, $\mathrm{H}_{2} \mathrm{O}_{2}$ exhibited an insensitive inhibitory effect on mycelial growth compared with $\mathrm{CdCl}_{2}$ and $\mathrm{NaCl}$, and the mycelial morphology under the $\mathrm{H}_{2} \mathrm{O}_{2}$ stress was similar with the control (Fig. 2a). These results were in accordance with the studies of Zhang et al. (2011) and Zharare et al. (2010), which showed that the mycelial growth and biomass of $\mathrm{H}$. marmoreus are significantly decreased with addition of $\mathrm{CdCl}_{2}, \mathrm{NaCl}$ or $\mathrm{H}_{2} \mathrm{O}_{2}$, and $\mathrm{CdCl}_{2}$ had a greater harmful effect than $\mathrm{NaCl}$ and $\mathrm{H}_{2} \mathrm{O}_{2}$.

Besides, the levels of $\mathrm{H}_{2} \mathrm{O}_{2}$ (Fig. 5a, c) and $\mathrm{O}_{2}{ }^{-}$(Fig. 5a) were also enhanced by these three pretreatments. Under most conditions, $\mathrm{ROS}\left(\mathrm{H}_{2} \mathrm{O}_{2}\right.$ and $\left.\mathrm{O}_{2}{ }^{-}\right)$can be efficiently scavenged by antioxidants, such as SOD, CAT and GR (Xie et al. 2012; Zhang et al. 2016b). In our study, pretreatments of $\mathrm{CdCl}_{2}, \mathrm{NaCl}$ and $\mathrm{H}_{2} \mathrm{O}_{2}$ significantly decreased the CAT and GR activities in mycelia, whereas the SOD activity was induced by the three pretreatments, which were consistent with the results of gene expression (Fig. 7). In mushrooms, antioxidants exhibit different responses to different stresses (Jiang et al. 2015; Zhang et al. 2016a). These results suggested that the high level of $\mathrm{H}_{2} \mathrm{O}_{2}$ or $\mathrm{O}_{2}{ }^{-}$in $\mathrm{CdCl}_{2}, \mathrm{NaCl}$ and $\mathrm{H}_{2} \mathrm{O}_{2}$-stressed mycelia was caused, at least in part, by an impaired detoxifying capacity of ROS. Similar with plants, some abiotic stresses, including heavy metals or salinity, can directly or indirectly cause damages to mushrooms by ROS production, which can induce lipid peroxidation and antioxidant responses in plants or mushrooms (Cao et al. 2012; Maria and Bebianno 2011). Our study also demonstrated that the pretreatments with $\mathrm{CdCl}_{2}, \mathrm{NaCl}$ and $\mathrm{H}_{2} \mathrm{O}_{2}$ enhanced MDA levels in mycelia of $H$. marmoreus (Fig. $5 \mathrm{~b}$ ), which is an index of lipid peroxidation and oxidative stress.

In plants, HRW has been found to enhance the antioxidant capacities of inducing plant tolerance to some stresses, such as salinity, $\mathrm{CdCl}_{2}$ and blue light-induced oxidative stress (Cui et al. 2013; Xie et al. 2012; Zhang et al. 2015c). In fungi, there is no report about the effects of HRW on the growth and antioxidant capacity. Our results demonstrated that HRW treatment ameliorated the adverse effects of $\mathrm{CdCl}_{2}, \mathrm{NaCl}$ and $\mathrm{H}_{2} \mathrm{O}_{2}$ in the mycelia of $H$. marmoreus. First, we found that the mycelia after the HRW treatment for 5 days were more quickly regenerated than the control group (Fig. 2a). Figure 2b and $\mathrm{c}$ reveal that the mycelial growth was enhanced and the mycelial biomass was increased after the HRW treatment. Besides, the $\mathrm{CdCl}_{2}, \mathrm{NaCl}$ and $\mathrm{H}_{2} \mathrm{O}_{2}$ accumulation in mycelia were attenuated after HRW treatment (Table 1). In plants, HRW can also enhance the growth response to different toxic factors, such as $\mathrm{CdCl}_{2}$ and $\mathrm{NaCl}$ (Cui et al. 2013; Xie et al. 2012; Zhang et al. 2015a). Our results indicated that HRW could effectively alleviate the growth inhibition and oxidative damage triggered by $\mathrm{CdCl}_{2}, \mathrm{NaCl}$ and $\mathrm{H}_{2} \mathrm{O}_{2}$ stresses in mycelia of H. marmoreus, which was in accordant with the previous study in plants.

Second, we found that HRW could enhance the antioxidant capacity of the mycelia in $H$. marmoreus. The HRW treatment led to a decrease in oxidative injuries caused by $\mathrm{CdCl}_{2}, \mathrm{NaCl}$ and $\mathrm{H}_{2} \mathrm{O}_{2}$, and the level of MDA was decreased (Fig. 5c). This finding was in good agreement with the reduced accumulation of $\operatorname{ROS}\left(\mathrm{H}_{2} \mathrm{O}_{2}\right.$ and $\mathrm{O}_{2}{ }^{-}$) and increased activities of antioxidants (SOD, CAT and GR). Besides, the HRW treatment also induced the expressions of antioxidants (SOD, CAT and GR) at the mRNA level. These results suggested that HRW might decrease lipid peroxidation in mycelia under these three stresses by activating antioxidants. In plants, such as rice (Xu et al. 2013), Arabidopsis (Xie et al. 2012) and Chinese cabbage (Wu et al. 2015a), HRW has the similar effects. One possible role of HRW might be that $\mathrm{H}_{2}$ can readily permeate the cell membrane, thereby increasing the gene expression of antioxidants (Cui et al. 2013), which is 


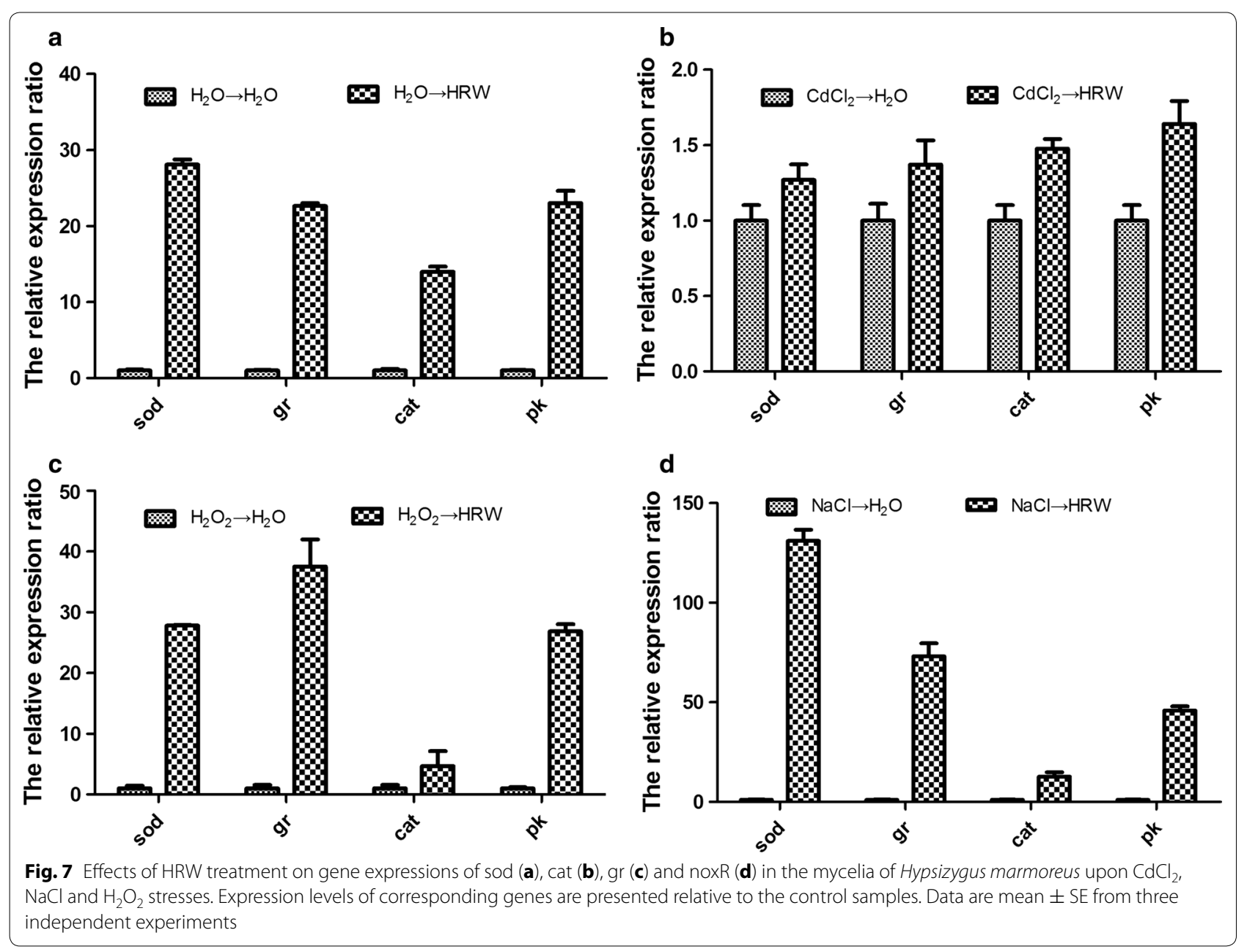

similar with our data. It has been observed in an in vitro experiment that $\mathrm{HRW}$ is able to directly quench $\mathrm{H}_{2} \mathrm{O}_{2}$, but not singlet oxygen radical (Xie et al. 2012). However, HRW could effectively scavenge $\mathrm{H}_{2} \mathrm{O}_{2}$ and $\mathrm{O}_{2}{ }^{-}$in the mycelia of H. marmoreus (Fig. 5).

In addition, HRW treatment could also enhance the PK activity in mycelia of $H$. marmoreus (Fig. 6a), which is a critical enzyme in glycolytic pathway. This result was in a good agreement with the increased expression of PK at the mRNA level (Fig. 6b). It has long been recognized that energy metabolism is linked to the production of ROS, and critical enzymes allied to metabolic pathways can be affected by redox reactions (Quijano et al. 2016). Mitochondria are primary sites of intracellular formation and reaction of ROS during the glucose metabolism (Brookes et al. 2004; Vasquez-Vivar et al. 2000). Lyngbya sp. can produce $\mathrm{H}_{2}$ during this metabolism (Shi and $\mathrm{Yu}$ 2016). In rice, HRW can activate $\alpha / \beta$-amylase activity, thus accelerating the formation of reducing sugar and total soluble sugar (Xu et al. 2013). These results suggested that HRW regulated the level of ROS to enhance the metabolic efficiency of sugar and provided more energy for mycelial growth.

In conclusion, we found that hydrogen-rich water (HRW) treatment could alleviate the toxicities of $\mathrm{CdCl}_{2}$, $\mathrm{NaCl}$ and $\mathrm{H}_{2} \mathrm{O}_{2}$, leading to improved mycelial growth and biomass. The HRW treatment decreased the levels of malondialdehyde (MDA) and ROS and significantly increased the activities of antioxidants (SOD, CAT and GR). These results suggested that HRW treatment might enhance the antioxidant abilities to induce the mycelial growth in $H$. marmoreus. Besides, pyruvate kinase was activated by HRW treatment, suggesting that HRW treatment also activated the glucose metabolism. These results suggested that the usage of HRW could be an effective approach for contaminant detoxification in $H$. marmoreus.

\section{Additional file}

Additional file 1: Table S1. Primer sets used for quantitative real-time PCR. 


\section{Abbreviations}

HRW: hydrogen-rich water; ROS: reactive oxygen species; MDA: malondialdehyde; SOD: superoxide dismutase; CAT: catalase; GPX: glutathione peroxidase; POD: guaiacol peroxidase; PK: pyruvate kinase; DAB: 3, 3, 9-diaminobenzidine; NBT: nitroblue tetrazolium.

\section{Authors' contributions}

JJZ, HC and ZYF conceived the study. JJZ, HC and HBH designed and performed most of the experiments. JJZ wrote the manuscript; MJC, ZYF and HW edited the manuscript. All authors read and approved the final manuscript.

\section{Author details}

${ }^{1}$ National Research Center for Edible Fungi Biotechnology and Engineering, Key Laboratory of Applied Mycological Resources and Utilization, Ministry of Agriculture, Shanghai Key Laboratory of Agricultural Genetics and Breeding, Institute of Edible Fungi, Shanghai Academy of Agricultural Sciences, No. 1000, Jinqi Road, Fengxian District, Shanghai 201403, China. ${ }^{2}$ College of Life Science, Nanjing Agricultural University, No.1, Weigang Road, Xuanwu District, Nanjing 210095, China.

\section{Acknowledgements}

We thank Jing Zhao for giving some helps in performing the experiments.

\section{Competing interests}

The authors declare that they have no competing interests.

\section{Availability of data and materials}

The dataset supporting the conclusions of this article is included within the article and its supplementary files.

\section{Funding}

This work was supported by grants from the National Natural Science Foundation of China (Grant Nos. 31601802 and 31401932), the Youth Talent Development Plan of Shanghai Municipal Agricultural System of China (Grant No. 20160111) and the Shanghai Municipal Agricultural Commission Development Program of China (Grant No. 20130510)

\section{Publisher's Note}

Springer Nature remains neutral with regard to jurisdictional claims in published maps and institutional affiliations.

Received: 7 March 2017 Accepted: 18 May 2017

Published online: 30 May 2017

\section{References}

Akavia E, Wasser SP, Beharav A, Nevo E (2006) Study of Hypsizygus marmoreus (Peck) Bigel. and Grifola frondosa (Dicks. Fr.) SF Gray: cultural-morphological peculiarities, growth characteristics, qualitative enzymatic activity, and resistance to fungal pest contamination. Int J Med Mushrooms 8:361-376

Bailly C (2004) Active oxygen species and antioxidants in seed biology. Seed Sci Res 14:93-107

Brookes PS, Yoon Y, Robotham JL, Anders MW, Sheu SS (2004) Calcium, ATP, and ROS: a mitochondrial love-hate triangle. Am J Physiol Cell Physiol 287(4):817-833

Cao YR, Zhang XY, Deng JY, Zhao QQ, Xu H (2012) Lead and cadmium-induced oxidative stress impacting mycelial growth of Oudemansiella radicata in liquid medium alleviated by microbial siderophores. World J Microbiol Biotechnol 28:1727-1737

Cui WT, Gao CY, Fang P, Lin GQ, Shen WB (2013) Alleviation of cadmium toxicity in Medica gosativa by hydrogen-rich water. J Hazard Mater 260:715-724

Hatvani N, Mecs I (2003) Effects of certain heavy metals on the growth, dye decolorization, and enzyme activity of Lentinula edodes. Ecotox Environ Safe 55:199-203

Huang CS, Kawamura T, Toyoda Y, Nakao A (2010) Recent advances in hydrogen research as a therapeutic medical gas. Free Radic Res 44:971-982

Järup L, Åkesson A (2009) Current status of cadmium as an environmental health problem. Toxicol Appl Pharmacol 238:201-208
Jiang J, Liu HY, Li Q, Gao N, Yao Y, Xu Y (2015) Combined remediation of Cdphenanthrene co-contaminated soil by Pleurotus cornucopiae and Bacillus thuringiensis FQ1 and the antioxidant responses in Pleurotus cornucopiae. Ecotoxicol Environ Safety 120:386-393

Jin QJ, Zhu KK, Cui WT, Xie YJ, Han B, Shen WB (2013) Hydrogen gas acts as a novel bioactive molecule in enhancing plant tolerance to paraquatinduced oxidative stress via the modulation of heme oxygenase-1 signalling system. Plant Cell Environ 36:956-969

Liu J, Zhou Q, Sun T, Ma L, Wang S (2008) Growth responses of three ornamental plants to $\mathrm{Cd}$ and $\mathrm{Cd}$-Pb stress and their metal accumulation characteristics. J Hazard Mater 151:261-267

Livak KJ, Schmittgen TD (2001) Analysis of relative gene expression data using real-time quantitative PCR and the 2(-Delta Delta C(T)) method. Methods 25:402-408

Maria VL, Bebianno MJ (2011) Antioxidant and lipid peroxidation responses in Mytilus galloprovincialis exposed to mixtures of benzo(a)pyrene and copper. Comp Biochem Physiol C Toxicol Pharmacol 154:56-63

Ohta S (2012) Molecular hydrogen is a novel antioxidant to efficiently reduce oxidative stress with potential for the improvement of mitochondrial diseases. Biochim Biophys Acta 1820:586-594

Podazza G, Arias M, Prado FE (2012) Cadmium accumulation and strategies to avoid its toxicity in roots of the citrus rootstock Citrumelo. J Hazard Mater 215:83-89

Quijano C, Trujillo M, Castro L, Trostchansky A (2016) Interplay between oxidant species and energy metabolism. Redox Biol 8:28-42

Rodríguez-Serrano M, Romero-Puertas MC, Zabalza A, Corpas FJ, Gómez M, Del Río LA, Sandalio LM (2006) Cadmium effect on oxidative metabolism of pea (Pisum sativum L.) roots. Imaging of reactive oxygen species and nitric oxide accumulation in vivo. Plant Cell Environ 29:1532-1544

Rodríguez-Serrano M, Romero-Puertas MC, Sparkes I, Hawes C, del Río LA, Sandalio LM (2009) Peroxisome dynamics in Arabidopsis plants under oxidative stress induced by cadmium. Free Radic Biol Med 47:1632-1639

Schützendübel A, Schwanz P, Teichmann T, Gross K, Langenfeld-Heyser R, Godbold DL, Polle A (2001) Cadmium-induced changes in antioxidative systems, hydrogen peroxide content, and differentiation in Scots pine roots. Plant Physiol 127:887-898

Shi XY, Yu HQ (2016) Simultaneous metabolism of benzoate and photobiological hydrogen production by Lyngbya sp. Renew Energy 95:474-477

Vasquez-Vivar J, Kalyanaraman B, Kennedy MC (2000) Mitochondrial aconitaseis a source of hydroxyl radical. An electron spin resonance investigation. J Biol Chem 275(19):14064-14069

Wu Q, Su NN, Cai JT, Shen ZG, Cui J (2015a) Hydrogen-rich water enhances cadmium tolerance in Chinese cabbage by reducing cadmium uptake and increasing antioxidant capacities. J Plant Physiol 175:174-182

Wu Q, Su NN, Chen Q, Shen WB, Shen ZG, Xia Y, Cui J (2015b) CadmiumInduced Hydrogen Accumulation is involved in cadmium tolerance in Brassica campestris by reestablishment of reduced glutathione homeostasis. PLoS ONE 10(10):e0139956

Xie YJ, Mao Y, Lai DW, Zhang W, Shen WB (2012) $\mathrm{H}_{2}$ enhances Arabidopsis salt tolerance by manipulating ZAT10/12-mediated antioxidant defence and controlling sodium exclusion. PLOS ONE 7:e49800

Xu S, Zhu SS, Jiang YL, Wang N, Wang R, Shen WB, Yang J (2013) Hydrogenrich water alleviates salt stress in rice during seed germination. Plant Soil 370:47-57

Zhang Z, Rengel Z, Meney K, Pantelic L, Tomanovic R (2011) Polynucleararomatic hydrocarbons (PAHs) mediate cadmium toxicity to an emergent wetland species. J Hazard Mater 189:119-126

Zhang JJ, Shi L, Chen H, Sun YQ, Zhao MW, Ren A, Wang H, Feng ZY (2014) An efficient Agrobacterium-mediated transformation method for the edible mushroom Hypsizygus marmoreus. Microbiol Res 169:741-749

Zhang XN, Zhao XQ, Wang ZQ, Shen WB, Xu XM (2015a) Protective effects of hydrogen-rich water on the photosynthetic apparatus of maize seedlings (Zea mays L.) as a result of an increase in antioxidant enzyme activities under high light stress. Plant Growth Regul 77:43-56

Zhang JJ, Chen H, Chen MJ, Ren A, Huang JC, Wang H, Zhao MW, Feng ZY (2015b) Cloning and functional analysis of a laccase gene during fruiting body formation in Hypsizygus marmoreus. Microbiol Res 179:54-63

Zhang JJ, Ren A, Chen H, Zhao MW, Shi L, Chen MJ, Wang H, Feng ZY (2015C) Transcriptome analysis and its application in identifying genes associated with fruiting body development in basidiomycete Hypsizygus marmoreus. PLOS ONE 10(4):e0123025 
Zhang JJ, Chen H, Chen MJ, Wang H, Song XX, Feng ZY (2016a) Construction and application of a gene silencing system using a dual promoter silencing vector in Hypsizygus marmoreus. J Basic Microbiol 56:1-9

Zhang SM, Zhang XL, Chang C, Yuan ZY, Wang T, Zhao Y, Yang XT, Zhang YT, La GX, Wu K, Zhang ZM, Li XZ (2016b) Improvement of tolerance to lead by filamentous fungus Pleurotus ostreatus $\mathrm{HAU}-2$ and its oxidative responses. Chemosphere 150:33-39
Zharare GE, Kabanda SM, Poku JZ (2010) Effects of temperature and hydrogen peroxide on mycelial growth of eight Pleurotus strains. Sci Hortic 125:95-102
Submit your manuscript to a SpringerOpen ${ }^{\circ}$ journal and benefit from:

- Convenient online submission

- Rigorous peer review

Immediate publication on acceptance

- Open access: articles freely available online

- High visibility within the field

- Retaining the copyright to your article

Submit your next manuscript at springeropen.com 